\title{
Das homogene Gravitationsfeld und die Lorentztransformationen.
}

Von Karl Bollert in Berlin-Friedenau.

Mit zwei Abbildungen. (Eingegangen am 4. Juni 1922.)

Der Zusammenhang zwischen der speziellen und der allgemeinen Relativitätstheorie ist dadurch hergestellt, $d a ß$ es in einem beliebigen Gravitationsfeld für die Umgebung eines jeden Weltpunktes lokale starre Bezugssysteme gibt, in denen die spezielle Relativitätstheorie gilt. Es sind das diejenigen infinitesimalen Bezugssysteme, welche frei beweglich sind, und daher an der Gravitationsbewegung teilnehmen. In bezug anf diese ist für alle freibeweglichen Körper, deren Massen so klein sind, daß ihre Rückwirkung anf das Feld ver-. nachlässigt werden kann, die Gravitationsbeschleunigung in der Umgebung des Weltpunktes gleich Null. In einem solchen lokalen Inertialsystem ist also kein Gravitationsfeld mehr vorhanden. Freibewegliche Körper bewegen sich nach dem Trägheitsgesetz, Uhren und Maßstäbe verhalten sich den Gesetzen der speziellen Relativitätstheorie entsprechend. Die relativ zu dem ursprünglichen Gravitationsfeld ruhenden Gegenstände, hängende Lampen usw., führen eine der Gravitationsrichtung entgegengesetzte beschleunigte Bewegung aus, deren Ursache von einem in dem Inertialsystem ruhenden Beobachter in den elastischen Spannungen der Aufhängevorrichtung gefunden wird.

Die lokalen Inertialsysteme haben in einem beliebigen Gravitationsfeld, der von Weltpunkt zu Weltpunkt variierenden Beschaffenheit des Feldes entsprechend, selbst eine relative Beschleunigung in bezug aufeinander: Es ist also im allgemeinen nicht möglich für einen endlichen Bereich das Gravitationsfeld fortzatransformieren. Unter einem homogenen Gravitationsfeld soll ein solches verstanden werden, dessen Feldwirkung für seine ganze Ausdehnung, von einem geeigneten starren System aus betrachtet, verschwindet. Nach der klassischen Mechanik würden in diesem starren Inertialsystem alle in dem ursprünglichen Gravitationsfeld ruhenden Körper gleiche, von Ort und Zeit unabhängige Bescbleunigungen haben. $\mathrm{Da}$ in dem Inertialsystem die spezielle Relativitätstheorie gelten soll, ist, wie wir gleich sehen werden, ein solches Verbalten unmöglich. Wir wollen uns die Verhältnisse in dem Inertialsystem anschanlich vor Augen führen, indem wir nach dem Beispiel von Minkowski uns auf die Vorgänge in einer Ebene beschränken. Wir erbalten dadurch die Möglichkeit, uns die aufeinanderfolgenden zeitlichen Zustände durch Ubereinander- 
schichten im dreidimensionalen Raume graphisch darzustellen (Fig. 1). Wir wollen das Inertialsystem so wählen, daß es zu Beginn der Zeitzählung mit dem Ruhsystem zusammenfällt. Die Weltlinien von freibeweglichen Massenpunkten, die zur Zeit $t=0$ relativ zu dem Gravitationssystem ruhten, sind dann zur $t$-Achse parallele Gerade, and mit ihnen verbundene objektiv gleiche Uhren, die zu Beginn

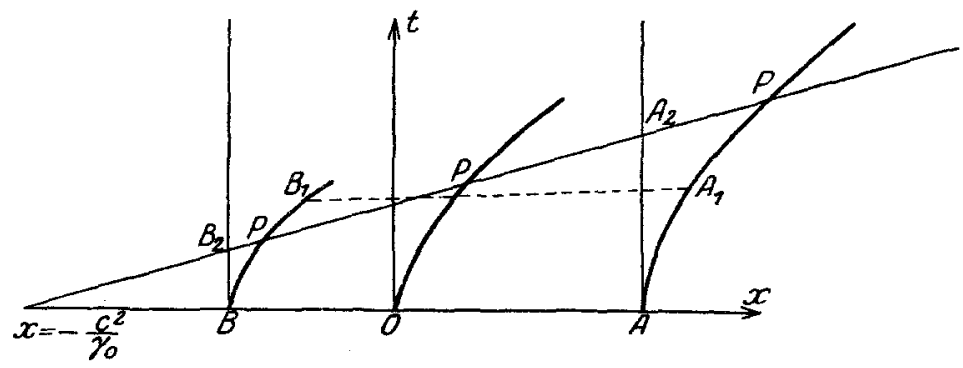

Fig. 1.

der Zeitzählung die gleiche Zeigerstellnng hatten, werden in jeder zur $t$-Achse senkrechten Ebene wieder eine gleiche Zeigerstellung aufweisen. Die Weltlinien der relativ zum Gravitationsfeld rahenden Gegenstände würden nach der klassischen Mechanik Parabeln sein, deren Gleichungen in dem Minkowskischen Körper die Form

$$
s=\left(x-x_{0}\right)=1 / 2 \gamma t^{2}
$$

besitzen würden, wo $\gamma$ eine konstante Größe ist.

Für beliebige Werte von $t$ ist das in der speziellen Relativitätstheorie ohne weiteres ausgeschloseen, da dann die Geschwindigkeit dieser materiellen Punkte $P$ über jede Grenze hinauswachsen würde. Aber auch für hinreichend kleine $t$ können die Bewegungsgleichnngen nicht die el wähnte Form mit konstantem $\gamma$ besitzen, denn das würde bedeuten, daß die Ruhbeschleunigung der starr miteinander verbundenen Punkte der $x$-Achse konstaut ist. Ibre Weltlinien würden also, wie Herr M. Born ermittelt hat, kongruente Hyperbeln sein, und die Länge eines starren, in der Bewegungsrichtung liegenden Stabes wäre für den Beobachter im Inertialsystem unabhängig von seiner Geschwindigkeit. Ein solcher Stab $A B$ von der Ruhlänge $d x$ muß aber, wenn er die Geschwindigkeit $v$ erlangt hat, die Lorentzverkürzung

$$
d x\left(1-\sqrt{1-\frac{v^{2}}{c^{2}}}\right)
$$

aufweisen. Dies ist offenbar nur möglich, wenn sein für den Inertialbeobachter vorderes Ende in derselben Zeit einen kleineren Weg 
zurücklegt als sein hinteres Ende. Bezeichnen wir die Koordinate seines vorderen Endes $A$ zu Beginn der Bewegung mit $x_{a}$, seines hinteren Endes $B$ mit $x_{b}$, die Geschwindigkeiten entsprechend mit $v_{a}$ und $v_{b}$, wo $v_{a}$ and $v_{b}$ nicht uar Funktionen von $t$ sondern auch von $x_{\alpha}$ beziehungsweise von $x_{b}$ sind, zwischen denen für dasselbe $t$ die Beziehung besteht $v_{a}=v_{b}+\frac{\partial v_{b}}{\partial x} d x$, so ist hiernach die Lorentzkontraktion

$$
\begin{gathered}
s_{b}-s_{a}=\int_{0}^{t} v_{b} d t-\int_{0}^{t} v_{a} d t \quad \text { oder } \\
-\int_{0}^{t} \frac{\partial v_{b}}{\partial x} d x d t=d x\left(1-\sqrt{1-\frac{v^{2}}{c^{2}}}\right)
\end{gathered}
$$

Durch Division von $d x$ und Differentiation nach $t$ ergibt sich

$$
-\frac{\partial v}{\partial x}=\frac{\partial\left(1-\sqrt{1-\frac{v^{2}}{c^{2}}}\right)}{\partial t}=\frac{\frac{v}{c^{2}} \frac{\partial v}{\partial t}}{\sqrt{1-\frac{v^{2}}{c^{2}}}} .
$$

Durch nochmalige Differentiation nach $t$ unter Vertauschnng der Differentiationsfolge auf der linken Seite folgt daraus

$$
-\frac{\partial \frac{\partial v}{\partial t}}{\partial x}=\frac{\partial^{g} v}{\partial t^{2}} \frac{v}{c \sqrt{c^{2}-v^{2}}}+\frac{\partial v}{\partial t} \frac{\partial \frac{v}{c \sqrt{c^{2}-v^{2}}}}{\partial t} .
$$

Für den langsam bewegten Punkt ergibt sich

$$
-\frac{\partial \dot{v}}{\partial x}=\frac{\dot{v}^{2}}{c^{2}} \text { oder }-\frac{\partial v}{\dot{v}^{2}}=\frac{\partial x}{c^{2}} .
$$

Die Integration ergibt $\frac{1}{\dot{v}}=\frac{x}{c^{2}}+C$. Für $x=0$ finden wir $C=\frac{1}{\dot{v}_{0}}$.

Es besteht also zwischen der Ruhbeschleunigung im Koordinatenanfangspunkt $\gamma_{0}$ und der Ruhbeschleunigung $\gamma$ in der Entfernung $x$ die Beziehung

$$
\gamma=\frac{\gamma_{0}}{1+\frac{\gamma_{0} x}{c^{2}}} .
$$

Da es sich nur um die Ermittlung der Ruhbeschleunigung handelt, hätten wir die Rechunng einfacher gestalten können, wenn wir, unter Berücksichtigung der Tatsache, daß $\gamma$ eine Funktion von $x$ ist, von der Gleichung (1) ansgegangen wären. 
Es wäre dann $s=\frac{\gamma}{2} t^{2}$ und

$$
\begin{aligned}
& s_{b}-s_{a}=\frac{\gamma}{2} t^{2}-\left(\frac{\gamma+d \gamma}{2}\right) t^{2}=d x\left(1-\sqrt{1-\frac{v^{2}}{c^{2}}}\right) . \\
& \text { Also } \frac{d \gamma t^{2}}{d x 2}=1-\sqrt{1-\frac{v^{2}}{c^{2}}}=\frac{\frac{v^{2}}{c^{2}}}{1+\sqrt{1-\frac{v^{2}}{c^{2}}}} .
\end{aligned}
$$

Setzen wir $v=\gamma t$, so ergibt sich für $t=0$

$$
-\frac{d \gamma}{d x}=\frac{\gamma^{2}}{c^{2}}
$$

in Ubereinstimmung mit der Gleichung oben. Setzen wir also in (1) für $\gamma$ den Wert (2) ein, so ist (1) für kleine $t$ die Gleichung eines der starr verbundenen im Gravitationsfeld ruhenden Punkte der $x$-Achse.

Die zweite Aufgabe, die wir zu lösen haben, ist die Bestimmung der Zeitfläche für den im Gravitationsfeld befindlichen Beobachter, d. h. die Ermittlung des Ortes aller derjenigen Weltpunkte, die für diesen Beobachter gleichzeitige Ereignisse darstellen. Atch hierfür liefert uns die spezielle Relativitätstheorie die notwendigen Grundlagen. Der eigentliche Gehalt der Lorentztransformationen liegt in der Verschiedenheit der Zeitmessung für zwei Beobachter, die relativ zneinander eine Geschwindigkeit haben. Lägen für beide die gleichzeitigen Ereignisse auf derselben Zeitebene, so würde ein Lichtkegel, der vor dem Koinzidenzpunkt der beiden Beobachter sich ausbreitete, von der Zeitebene in Kreisen geschnitten werden, die für den bewegten Beobachter exzentrisch sind. Dieser wïrde sich also nicht im Mittelpunkt der Kagelwellen befinden, sondern die Lichtgeschwindigkeit würde in irgend einem Punkte der $x$-Achse in der positiven Richtung eine andere sein als in der negativen. Die Zeitebene muß deshalb für den bewegten Beobachter so geneigt sein, daß auch dieser sich dauernd im Mittelpunkt der Lichtwelle befindet. Dieses Axiom von der Umkehrbarkeit des Lichtweges, welches schon der speziellen Relativitätstheorie zugrunde liegt, wollen wir in die allgemeine mit herübernehmen. Um die Zeitfläche für den Gravitationsbeobachter zu erhalten, müssen wir also durch den Raam-Zeit-Körper diejenige Fläche legen, die aus allen infinitesimalen Lichtkegeln Ellipsen herausschneidet, deren Mittelpunkte auf den durch die Scheitelpunkte gehenden Weltlinien der im Gravitationssystem rubenden materiellen Punkten $P$ liegen. Die Gleichungen dieser materiellen Pankte sind zu Beginn der Bewegung dargestellt darch die Gleichungen (1). Die 
Tangenten் dieser Karven sind für den Gravitationsbeobachter die momentanen Zeitachsen. In der Umgebung eines im Gravitationssystem ruhenden Weltpunktes müssen also die von diesem Punkt ausgehenden beiden Seitenlinien des Lichtkegels zwei zugeordnete, die Tangente in diesem Punkt und die in der Zeichenebene liegende Spur der

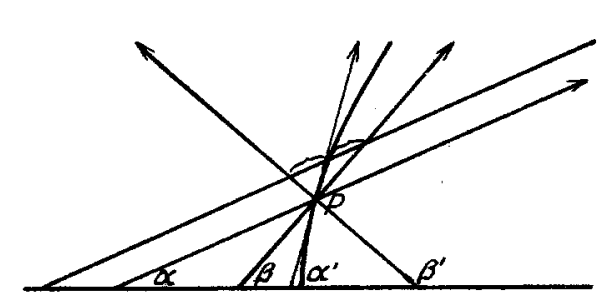

Fig. 2. Zeittläche die beiden anderen zugeordneten Strahlen eines harmonischem Büschels bilden. Das sind offenbar die Bedingungen, daß das zwischen den beiden Seitenlinien liegende Stück einer zur Spur parallelen Strecke von der Tangente halbiert wird.

Bezeichnen wir die Richtungswinkel der 4 durch $P(x t)$ gehenden harmonischen Strahlen wie in Fig. 2, so ist der analytische Angdruck für diese Forderung:

$$
\operatorname{tg} \alpha \operatorname{tg} \alpha_{1}+\operatorname{tg} \beta \operatorname{tg} \beta_{1}=0
$$

Von diesen Richtangskoeffizienten $\frac{d t}{d x} \operatorname{sind} \operatorname{tg} \beta$ und $\operatorname{tg} \beta_{1}$ bekannt als die reziproken Werte der Geschwindigkeit des Lichtes in der positiven und negativen Richtung der $x$-Achse und $\operatorname{tg} \alpha_{1}$ nach Gleichung (1) als die Geschwindigkeit des Punktes P. Es ist also

$$
\operatorname{tg} \beta=\frac{1}{c}, \quad \operatorname{tg} \beta_{1}=-\frac{1}{c}, \quad \operatorname{tg} \alpha_{1}=\frac{1+\frac{\gamma_{0} x}{c^{2}}}{\gamma_{0} t} \begin{aligned}
& \text { (Für kleine } \\
& \text { Werte von } t \text { ). }
\end{aligned}
$$

Die Differentialgleichung unserer Zeitfläche lautet also

$$
\frac{d t}{d x} \frac{1+\frac{\gamma_{0} x}{c^{2}}}{\gamma_{0}}-\frac{1}{c^{2}}=0
$$

Die Integration ergibt $\ln t=\ln \left(1+\frac{\gamma_{0} x}{c^{2}}\right)+C$, für $x=0$ erhalten wir $\ln t_{0}=C$.

Die Gleichung der Zeitfläche lautet also für kleine Werte von $t$

$$
t=t_{0}\left(1+\frac{\gamma_{0} x}{c^{2}}\right) \text {. }
$$

Unter denselben Voraussetzungen sind $t$ und $t_{0}$ nicht verschieden von den Eigenzeiten $d \tau$ der mit den Punkten $P$ verbundenen Uhren and es ist $x$ zugleich die mit starren Maßstäben im Gravitations- 
system gemessene Entfernung vom Anfangspunkt. In der Gleichung $d \tau=d \tau_{0}\left(1+\frac{\gamma_{0} x}{c^{2}}\right)$ sind also keine anf das ursprüngliche Inertialsystem sich beziehenden Größen mehr vorhanden. Sie ist also nicht mehr an den Zeitpunkt zu Beginn der Fallbewegung des von uns gerade gewählten Ruhesystems gebunden, sondern gilt, wie das bei den stationären Verhältnissen im Gravitationssystem auch selbstverständlich ist, ohne Einschränkung. Die Verhältnisse im Gravitationssystem gestalten sich jetzt sehr anschaulich. Bezogen auf das momentane Ruhesystem dreht sich die Zeitebene, wie aus Fig. 1 hervorgeht, um den Punkt $x=-\frac{e^{2}}{\gamma_{0}}$. Die der gravitierenden Masse näher liegenden Ereignisse verlaufen also langsamer. Die Gleichung tg $\alpha_{1}=\frac{1+\frac{\gamma_{0} x}{c^{2}}}{\gamma_{0} t}$ geht mit Benutzung von (3) über in $\operatorname{tg} \alpha_{1}=\frac{t_{0}}{\gamma_{0}}$. Die Weltlinien der Punkte $P$ werden also von der Zeitebene unter gleichem Winkel geschnitten. Sie bilden daher in bezug auf den Drehpunkt eine Schar ähnlicher und ähnlich liegender Karven. Die in unserer graphischen Darstellung in Fig. 1 vorhandene Dehnung der Abstände zweier Punkte $P$ ist also für den Gravitationsbeobachter nicht vorhanden, da seine Maßstäbe sich entsprechend vergrößern. In seinem Raum gilt die euklidische Geometrie. Die Invariante des homogenen Feldes ist nach allem also

$$
d s^{2}=c^{2}\left(1+\frac{\gamma_{0} x}{c^{2}}\right)^{2} d \tau_{0}^{2}-d x^{2}-d y^{2}-d z^{2} .
$$

Zum Schluß wollen wir noch das Fallgesetz für das Gravitationssystem angeben. Es lautet für den langsam bewegten Punkt $s=\frac{1}{2} \gamma_{x} t_{x}{ }^{2}$ oder unter Benutzung von 1 und 3:s $=\frac{1}{2} \gamma_{0} \tau_{0}^{2}\left(1+\frac{\gamma_{0} x}{c^{2}}\right)$. Die Ruhbeschleunigung ist also

$$
\frac{d^{2} s}{d \tau_{0}^{2}}=-\gamma_{0}\left(1+\frac{\gamma_{0} x}{c^{2}}\right)
$$

Diese Werte hätten wir äbrigens anch aus (4) erbalten können. Die Weltlinien von freibeweglichen materiellen Pankten $A_{2}$ and $B_{2}$ sind charakterisiert darch die Gleichung $\delta \int d s=0$, wo ist.

$$
d s=d \tau_{0} \sqrt{c^{2}\left(1+\frac{\gamma_{0} x}{c^{2}}\right)^{2}-\left(\frac{d x}{d \tau_{0}}\right)^{2}-\left(\frac{d y}{d \tau_{0}}\right)^{2}-\left(\frac{d z}{d \tau_{0}}\right)^{2}}=H d \tau_{0}
$$


Die erste der dazugehörigen Lagrangeschen Gleiohungen lautet $\frac{d \frac{\partial H}{\partial q_{x}}}{d \tau_{0}}-\frac{\partial H}{\partial x}=0 \quad$ oder $\quad \frac{d\left(-\frac{d x}{H d \tau_{0}}\right)}{d \tau_{0}}-\frac{\left(1+\frac{\gamma_{0} x}{c^{2}}\right) \gamma_{0}}{H}=0$

Für kleine Geschwindigkeiten $H=c\left(1+\frac{\gamma_{0} x}{c^{2}}\right)$ also

$$
\frac{d^{2} x}{d \tau_{0}^{2}}=-\left(1+\frac{\gamma_{0} x}{c^{2}}\right) \gamma_{0} .
$$

In Beginn hatten wir gefunden, daß ein beschleunigter starrer Stab die Lorentzverkürzung anfweist, wenn die Beschleunigung seines hinteren Endes das $\left(1+\frac{\gamma x}{c^{2}}\right)$ fache seines vorderen Endes beträgt. Für den Gravitationsbeobachter haben die Beschleunigungen des freifallenden Stabes $A_{2} B_{2}$ diese Eigenschaft, wenn wir bedenken, daß für ihn $A_{2}$ das hintere Ende ist (Fig. 1). Es ergibt sich also auch für ihn eine Lorentzkontraktion.

Bei der Benutzung von $\delta \int d s=0$ sind die Punkte des Stabes $A B$ so behandelt, als wenn jeder für sich fiele. Wir kommen also zn dem richtigen Fallgesetz (5), wenn wir annehmen, daß ein ausgedehnter Körper in einem homogenen Felde spannungsfrei fällt. Von hier ans fallt ein neues Licht auf die Entstehung der Lorentzkontraktion. Wir finden nämlich für den bewegten Körper dieselben Verhältnisse wie für den einzelnen Punkt. Daß ein solcher im Inertialfelde sich nach dem Trägheitsgesetz bewegt und im Gravitationsfelde nach den Gravitationsgesetzen, sind nicht zwei verschiedene Tatsachen, sondern ist nach dem Aquivalenzprinzip nur eine Tatsache, von zwei verschiedenen Bezugssystemen ans betrachtet. Genau so müssen bewegte starre Körper im Inertialsystem die Lorentzkontraktion aufweisen, damit solche im Gravitationssystem spannungsfrei fallen können und umgekehrt. Letzten Endes ist die Lorentzkontraktion, die einer von zwei relativ zueinander beschleunigten Stäben aufweist, auf die Natur der Spannungen zurückzuführen, die in einem von diesen Stäben immer vorhanden sein müssen, and zwar sind dies im Inertialsystem die elastischen Spannungen des beschleunigten Stabes und im Gravitationssystem die ihnen entsprechenden Gravitationsspannungen des im Felde rnhenden Bezugssystems. Denken wir uns als $x$-Achse einen mit den gravitierenden Massen fest verbundenen Stab von dem Querschnitt 1 und der homogenen Massendichte $m$, der über den Koordinatenanfangspunkt um die Länge $l$ hinausragen möge. Die für den Inertialbeobachter in ihm vorhandenen elastischen Spannungen lassen 
sich einfach berechnen, wenn wir $m$ so klein annehmen, daß die Rückwirkungen des Stabes auf das Feld nicht in Rechnung gesetzt zu werden brauchen. Die Spannungen im Pankte $x$ sind dann gleich dem Gewicht des Stabendes von der $l-x$ oder $\int_{x}^{l} m \gamma d x$. Wir erhalten die Spannungen nur richtig, wenn wir für $\gamma$ nicht den Wert 5, den $\gamma$ für den Gravitationsbeobachter annimmt, wenn er sich bei den Messungen seiner im Anfangspunkt befindlichen Uhr bedient, sondern den natürlich gemessenen Wert einführen. Unter diesem natürlich gemessenen Wert von $\gamma$ verstehen wir den Wert der Beschlounigung an einem Punkt, verglichen mit einer dort befindlichen Uhr, denn diese lokalen Uhren, die im Inertialsystem alle die gleiche Geschwindigkeit anzeigen, sind es, mit denen in diesem System die Zeit gemessen wird. Da $s=\frac{1}{2} \gamma_{x} t_{x}^{2}$ ist, ist die natürliche Beschleanigung

$$
\gamma_{x}=\frac{\gamma_{0}}{1+\frac{\gamma_{0} x}{c^{2}}}
$$

Wir haben also für die Größe der Spannungen, die der Inertialbeobachter an dem Stab feststellt, den Wert

$$
t_{x}=\int_{x}^{l} \frac{m \gamma_{0}}{1+\frac{\gamma_{0} x}{c^{2}}} d x=m c^{2} \ln \frac{\left(1+\frac{\gamma_{0} l}{c^{2}}\right)}{\left(1+\frac{\gamma_{0} x}{c^{2}}\right)}
$$

Hieraus ergibt sich wieder mit Hilfe des Impalssatzes 1)

der Ausdruck 2.

$$
-d i v t_{x}=\frac{d m v}{d t}=-\frac{\partial t_{x}}{\partial x}
$$

Man kann die Frage aufwerfen, wie sich denn ein solches Inertialsystem, wie wir es bei unseren Utberlegungen benutzt haben, im Prinzip realisieren läßt. Würde man die obere Verbindung einer im Felde hängenden Stange oder die untere einer anfgerichteten einfach lösen, so würde ein solches System sich erst Punkt für Pankt sukzessive entspannen, ehe es frei fällt. Es würde daher für unsere Zwecke nicht geeignet sein. Wir können uns aber etwa folgende Vorrichtung denken: auf einen starken, länglichen Körper mit großem Elastivitäts-

1) Aus der Drehung der Zeitebene in einem homogenen Gravitationsfelde ergibt sich auch, wie ich an anderer Stelle gezeigt habe (Die Einsteinsche Gravitationstheorie und ihre Stellung im System der Gesamterfahrung, S. 24 usw.), eine einfache Erklärung des Uhrenparadoxons. 
modul werde, während er senkrecht zum Felde, also in seiner Längsrichtang ungespannt ruht, eine Anzahl sehr dünner Leisten mit geringem Elastizitätsmodul in einem solchen Zustand der Dehnung geleimit, daß sie beim Aufrichten dieser Vorrichtung spannungsfrei sind. Eine solohe Leiste denken wir ans in ihrer ganzen Ausdehnung für den Gravitationsbeobachter gleichzeitig, also in den Weltpunkten $P$ gelöst. Da keine Ruhespannangen in diesen Leistungen vorhanden sind, sind auch in bewegtem Zustande in der Richtung der $x$-Achse keine vorhanden, denn diese Spannungen in der $x$-Richtung sind eine Invariante der Lorentztransformation. Nach Fig. 1 bewegen sich die Punkte $P$ auf den durch $P$ gelegten Tangenten, die nach Fig. 1 untereinander parallel sind. In der gleichen Weise sei auch schon das ursprüngliche Inertialsystem sowie eine Reihe weiterer hergestellt. Eine solche Vorrichtung kann also als Paradigma für die Beschleunigung eines Stabes, ohne seinen inneren Zustand $\mathrm{zu}$ ändern, dienen. Alle diese relativ zueinander bewegten Bezugssysteme weisen, wie wir im Vorhergehenden bewiesen haben, wenn die relative Geschwindigkeit zwischen zwei aufeinanderfolgenden klein ist, die Lorentzkontraktion anf. Dasselbe muß also auch für zwei in der Reihe beliebig anseinanderstehende Bezugssysteme gelten (nach dem Einsteinschen Additionstheorem von Geschwindigkeiten). Zusammenfassend können wir jetzt folgendes sagen: die Entstehung der Lorentzverkürzung ist kausal bedingt durch elastische Spannungen. Dafür aber, daß ein Stab, wenn er aus der beschleunigten Bewegung in die gleichförmige übergegangen ist, diese Lorentzverkürzang behält, läßt sich keine Ursache angeben, sondern nur einen Grund, und dieser Grund liegt in der verschiedenen Art der Zeitmessung in den beiden Systemen. Die fertige Kontraktion ist also nicht durch einen besonderen Spannungszustand ermöglicht, sondern phänomenologisch bedingt, genau wie man in der alten Physik dafür, daß im Euklidischen Raum ein Stab aus der Ruhelage in die Drehbewegung übergeht, eine Ursache angeben müßte, während es für die damit verbandene Anderung der Länge der Projektionen dieses Stabes in bezug anf irgendwelche Ebenen, nur einen mathematischen Grund gibt ${ }^{1}$ ).

Vergleich mit der allgemeinen Theorie. Nach der Einsteinschen Gravitationslehre sind die physikalischen Verhältnisse eines beliebigen Gravitationsfeldes bestimmt durch eine Grundinvariante von der Form $d s^{2}=\sum_{1}^{4} h g_{i k} d x_{i} d x_{k}$. Da das homogene Feld zu den

1) Über diesen Unterschied vergleịche 1. c., 8. 63. 
stationären gehört, müssen $g_{14}, g_{24}, g_{34}$ verschwinden und die übrigen $g_{i k}$ dürfen die Zeitkoordinate $d x_{4}$ nicht enthalten. Wählen wir das Koordinatensystem so, daß die Richtung der $x_{1}$-Achse mit der Richtung der Gravitationsbeschleunigung zusammenfällt und die $x_{2}$ - und $x_{3}$-Achse sowohl zur $x_{1}$-Achse wie zueinander senkrecht stehen, so sind die $g_{i k}$ nur noch Funktionen von $x_{1}$. Im anderen Fall würde nämlich eine Parallelverschiebung des Anfangspunktes in der $\left(x_{2} x_{3}\right)$ Ebene die Invariante ändern. Ein homogenes Feld ist aber dadurch charakterisiert, daß alle Punkte in einer solchen Ebene sich gleich verhalten. Ebenso wie gegen eine solche Verschiebung muß die Invariante einer Drehung des Bezugssystems um die $x$-Achse gegenüber unverändert bleiben. Die Invariante hat also notwendig die Form

$$
d s^{2}=g_{44} d x_{4}^{2}+g_{11} d x_{1}^{2}+g_{28}\left(d x_{2}^{2}+d x_{3}^{2}\right) .
$$

Da sie zuletzt auch durch eine Verschiebung auf der $x_{1}$-Achse wieder in einen Ausdruck von derselbeu Form übergehen muß, kann $g_{22}$ nur eine Konstante sein, die bei passender Wahl der Maßeinheiten von $d x_{2}$ und $d x_{3}$ gleich -1 gesetzt werden kann. Die Funktionen $g_{44}$ and $g_{11}$ sind nun so zu bestimmen, daß dem Einsteinschen Gravitationsgesetz genügt wird. Dieses lautet für den materiefreien Ranm:

$$
R_{k l}=\sum \frac{\partial \cdot\left\{\begin{array}{l}
k m \\
m
\end{array}\right\}}{\partial x_{l}}-\sum \frac{\partial\left\{\begin{array}{l}
k l \\
m
\end{array}\right\}}{\partial x_{m}}+\sum_{m n}\left\{\begin{array}{c}
k n \\
m
\end{array}\right\}\left\{\begin{array}{c}
{[m} \\
n\}
\end{array}\right\}-\left\{\begin{array}{c}
k l \\
m
\end{array}\right\}\left\{\begin{array}{c}
m n \\
n
\end{array}\right\}=0
$$

wo

$$
\left\{\begin{array}{c}
j k \\
l
\end{array}\right\}=\frac{1}{2} \sum_{i} g^{i l}\left(\frac{\partial g_{k i}}{\partial x_{j}}+\frac{\partial g_{i j}}{\partial x_{k}}-\frac{\partial g_{j k}}{\partial x_{i}}\right) .
$$

Wir wollen für $g_{44}$ den Wert $\nabla^{2}$ schreiben.

Die Determinante $g$ der quadratischen Form $d s^{2}$ ist dann nach dem vorigen

$$
\left|\begin{array}{rrrr}
g_{11} & 0 & 0 & 0 \\
0 & -1 & 0 & 0 \\
0 & 0 & -1 & 0 \\
0 & 0 & 0 & \nabla 2
\end{array}\right|
$$

Also $g=g_{11} V^{2}$. Die normierten Unterdeterminanten baben die Werte $g^{11}=\frac{1}{g_{11}}, \quad g^{22}=g^{33}=-1, \quad g^{44}=\frac{1}{\nabla^{2}} . \quad$ Es $\quad$ ist $\quad \frac{\partial g_{11}}{\partial x_{1}}=g_{11}^{\prime}$, $\frac{\partial g_{44}}{\partial x_{7}}=2 V V^{\prime}$, wäbrend die übrigen partiellen Differentialquotienten verschwinden: Von den Klammerausdrücken ist $\left\{\begin{array}{c}11 \\ 1\end{array}\right\}=\frac{g_{11}^{\prime}}{2 g_{11}}$, 
$\left\{\begin{array}{c}44 \\ 1\end{array}\right\}=-\frac{\nabla V^{\prime}}{g_{11}}$ and $\left\{\begin{array}{c}41 \\ 4\end{array}\right\}=\frac{\nabla^{\prime}}{\nabla}$, alle anderen sind gleich Null. Wir finden daher

und

$$
R_{11}=\frac{\partial \frac{V^{\prime}}{\nabla}}{\partial x_{1}}-\left(\frac{g_{11}^{\prime}}{2 g_{11}}-\frac{V^{\prime}}{V}\right) \frac{\nabla^{\prime}}{V}=0
$$

$$
R_{44}=\frac{\partial \frac{V \nabla^{\prime}}{g_{11}}}{\partial x_{1}}-\left(\frac{V^{\prime}}{\nabla}-\frac{g_{11}^{\prime}}{2 g_{11}}\right) \frac{V \nabla^{\prime}}{g_{11}}=0 .
$$

Alle anderen $\boldsymbol{R}_{i k}$ verschwinden identisch.

Entwickeln wir den Differentialquotienten, der in beiden Gleichungen das erste Glied bildet, so erweisen sich beide als identisch mit der Gleichung

$$
\nabla^{\prime \prime}-\frac{g_{11}^{\prime}}{2 g_{11}} \nabla^{\prime}=0
$$

Wir können also eine der Funktionen $g_{11}$ oder $\nabla$ willkürlich wählen. Setzen wir $g_{11}=-1$, so ergibt sich $V^{\prime \prime}=0$ oder $V=a x+b$. Diese Wahl würde, wie ein Blick anf $d s^{2}$ zeigt, bedeuten, daß $x$ physikalisch den natürlich gemessenen Abstand darstellt. Bei dieser Wabl der Variabeln lautet also die Grundinvariante

$$
d s^{2}=d x_{4}^{2}(a x+b)^{2}-\mathrm{d} x^{2}-d y^{2}-d z^{2} .
$$

Wenn man in geeigneter Weise über die Konstanten verfügt, ergibt sich also wieder unser Ausdruck 4. 